\title{
PERFIL DE MORTALIDADE MATERNA NA ÚLTIMA DÉCADA (2010 - 2019) NO ESTADO DA BAHIA
}

Profile of maternal mortality in the last decade $(2010$ - 2019) in the state of Bahia

Perfil de mortalidad materna en la última década (2010 - 2019) en el estado de Bahia

Ana Flávia Souto Figueiredo Nepomuceno • Mestranda do Programa de PósGraduação em Farmácia • Universidade Federal da Bahia • E-mail: ana.souto@ufba.br

Mariana Souto Figueiredo • Mestranda do Programa de Pós-Graduação em Saúde Coletiva • Universidade Federal da Bahia • E-mail: marianasoutofigueiredo@gmail.com

Veríssimo Santos de Jesus • Especialista em Saúde da Família • Fundação Estatal da Saúde da Família/ Fundação Oswaldo Cruz •

E-mail: verissimosantosdejesus@gmail.com

Liz Oliveira dos Santos • Professora Doutora $\bullet$ Universidade Federal do Recôncavo da Bahia・E-mail: liz@ufrb.edu.br

Autora correspondente:

Ana Flávia Souto Figueiredo Nepomuceno• E-mail: ana.souto@ufba.br 


\section{RESUMO}

Introdução: A mortalidade materna é um problema de saúde pública com elevada prevalência e que pode ser, na maioria dos casos, prevenida. As últimas décadas mostraram decréscimo nos índices, no entanto, nota-se que esse problema afeta principalmente regiões com maior vulnerabilidade social, dentre as quais destaca-se $o$ nordeste brasileiro onde encontra-se o estado da Bahia. Objetivo: Analisar o perfil de mortalidade materna na última década (2010 - 2019) no estado da Bahia. Metodologia: Trata-se de um estudo do tipo epidemiológico, transversal, retrospectivo e descritivo, tendo como base a análise secundária de informações disponíveis no Sistema de Informação sobre Mortalidade no período correspondente de 2010 a 2019 no estado da Bahia, Brasil. Resultados: No período estudado segundo o Sistema de Informação sobre Mortalidade, foram computados um total de 1313 óbitos maternos no estado da Bahia. A maioria das acometidas apresentava faixa etária de 30 a 39 anos $(44,35 \%)$, com maior prevalência de pessoas não brancas $(81,11 \%)$. Quanto à causa da morte, o estudo obteve predomínio de mortes por causas diretas $(66,61 \%)$ que ocorreram de modo majoritário em ambiente hospitalar $(90,17 \%)$. Conclusões: Observa-se por meio do presente estudo que a mortalidade materna na Bahia se constitui como um importante agravo em saúde, o que demanda a elaboração de um conjunto de medidas a fim de garantir segurança às gestantes e puérperas do estado.

Palavras-Chave: Gravidez, Mortalidade Materna, Morte, Saúde da Mulher.

\section{ABSTRACT}

Introduction: Maternal mortality is a public health problem with high prevalence and which can be prevented in most cases. The last decades have shown a decrease in the indexes, however, it is noted that this problem mainly affects regions with greater social vulnerability, among which the Northeast of Brazil stands out, where the state of Bahia is located. Objective: Analyze the profile of maternal mortality in the last decade (2010 - 2019) in the state of Bahia. Methodology: This is an epidemiological, cross-sectional, retrospective and descriptive study, based on the secondary analysis of information available in the Mortality Information System in the period corresponding to 2010 to 2019 in the state of Bahia, Brazil. Results: In the period studied, according to the Mortality Information System, a total of 1313 maternal deaths were computed in the state of Bahia. Most of those affected were aged 30 to 39 years $(44.35 \%)$, with a higher prevalence of non-white people $(81.11 \%)$. As for the cause of death, the study showed a predominance of deaths from direct causes $(66.61 \%)$, which pccurred mostly in hospitals $(90.17 \%)$. Conclusions: It is observed through this study that maternal mortality in Bahia constitutes an important health problem, which demands the elaboration of a set of measures in order to guarantee safety to pregnant omen and women in the state.

ywords: Death, Maternal Mortality, Pregnancy, Women's Health . 


\section{RESUMEN}

Introducción: La mortalidad materna es un problema de salud pública de alta prevalencia y que puede prevenirse en la mayoría de los casos. Las últimas décadas han mostrado una disminución en los índices, sin embargo, se nota que este problema afecta principalmente a regiones con mayor vulnerabilidad social, entre las que se destaca el Nordeste de Brasil, donde se ubica el estado de Bahia. Objetivo: Analizar el perfil de la mortalidad materna en la última década (2010 - 2019) en el estado de Bahía. Metodología: Se trata de un estudio epidemiológico, transversal, retrospectivo y descriptivo, basado en el análisis secundario de la información disponible en el Sistema de Información de Mortalidad (SIM) en el período correspondiente a 2010 a 2019 en el estado de Bahía, Brasil. Resultados: En el período estudiado, según el Sistema de Información de Mortalidad, se computaron un total de 1313 muertes maternas en el estado de Bahía. La mayoría de los afectados tenían entre 30 y 39 años $(44,35 \%)$, con mayor prevalencia de personas no blancas $(81,11 \%)$. En cuanto a la causa de la muerte, el estudio obtuvo un predominio de las muertes por causas directas $(66,61 \%)$, que se produjeron mayoritariamente en los hospitales $(90,17 \%)$. Conclusiones: Se observa a través de este estudio que la mortalidad materna en Bahía constituye un importante problema de salud, que exige la elaboración de un conjunto de medidas para garantizar la seguridad de las embarazadas y mujeres en el estado.

Palabras clave: Embarazo, Mortalidad Materna, Muerte, Salud de la Mujer. 


\section{Introdução}

Segundo a Organização Mundial da Saúde (OMS), a mortalidade materna pode ser conceituada como um evento trágico que pode ocorrer no decorrer do processo reprodutivo, que corresponde ao período durante a gestação ou de até 42 dias após o término dessa ${ }^{1}$. Por muito tempo, os óbitos decorrentes de complicações na gestação e puerpério foram considerados fatalidades, no entanto, atualmente, consiste em um importante agravo em saúde pública, tendo em vista sua elevada prevalência² ${ }^{2}$.

Estimativas apontam que, a cada minuto no mundo, uma mulher vai a óbito em decorrência de complicações durante a gestação ou no parto, trazendo à tona fragilidades nas condições de assistência, qualidade e, acesso das gestantes aos serviços de saúde oferecidos, uma vez que $90 \%$ desses óbitos poderiam ser evitados ${ }^{2,3}$.

Nas últimas décadas, em todo o mundo, notou-se uma tendência decrescente dos índices de mortalidade materna ${ }^{4,5}$. No Brasil, uma série de fatores contribuíram para esse decréscimo, dentre os quais apontam-se a implantação do Programa de Agentes Comunitários em 1991, e na mesma década do Programa de Saúde da Família que, posteriormente, no ano de 2006, deram lugar à Estratégia de Saúde da Família, com ênfase nas medidas de prevenção e recuperação aliadas ao cuidado longitudinal em saúde com base nas necessidades locais ${ }^{5}$. Ademais, houve um crescimento exponencial no acesso aos exames pré-natais 6 . No entanto, apesar desse notável progresso, os índices brasileiros ainda estão muito aquém aos propostos pela Organização das Nações Unidas7, sobretudo nas regiões de maior vulnerabilidade social como no Nordeste do país 4 .

No cenário de regiões com menores índices de desenvolvimento humano (IDH), destaca-se o estado da Bahia, que apresenta elevados índices de mortalidade materna ${ }^{8}$. Dessa forma, em razão da lacuna no conhecimento existente sobre o perfil de mulheres acometidas, a realização de estudos sobre a temática durante a última década é de fundamental importância para que as causas e fatores associados se ornem conhecidos, com o intuito de direcionar a abordagem dessas mulheres nas pas de planejamento familiar, gestação e o puerpério. Diante disso, este estudo tem ho objetivo analisar o perfil de mortalidade materna na última década (2010 - 2019) stado da Bahia. 


\section{Metodologia}

Trata-se de um estudo do tipo epidemiológico, transversal, retrospectivo e descritivo, tendo como base a análise secundária de informações disponíveis no Sistema de Informação sobre Mortalidade (SIM), no período correspondente a 2010 a 2019, no estado da Bahia. A coleta foi realizada no mês de novembro de 2020, e as causas de mortes maternas foram descritas de acordo com as categorias da $10^{\mathrm{a}}$ revisão da Classificação Internacional de Doenças (CID-10).

A análise dos dados foi feita mediante a utilização do software Microsoft Office Excel ${ }^{\circledR}$ onde os dados foram tabulados e posteriormente foram analisados estatisticamente no software Statistical Package for the Social Sciences® (SPSS), versão 21.0, onde foram analisadas as seguintes variáveis: totais de óbitos correspondentes, municípios com maior prevalência, raça e cor das vítimas, faixa etária, seguida pelas causas dos óbitos e o local de ocorrência. Foram excluídos dados que não contemplassem o período temporal estabelecido, bem como os que não pertencessem ao estado da Bahia.

Foi dispensada a submissão junto ao Comitê de Ética em Pesquisa pela utilização de dados de caráter secundário, não havendo identificação dos participantes do estudo.

\section{Resultados}

No período correspondente, foram computados um total de 1313 óbitos maternos no estado da Bahia, o que, a caráter nacional, representa aproximadamente 7,88\% do total de óbitos de gestantes e puérperas em todo o país entre 2010 a 2019. Os Municípios de Salvador (33,74\%), Feira de Santana (6,24\%) e Vitória da Conquista $(4,80 \%)$ apresentam-se como os municípios do Estado da Bahia com maior prevalência de mortes. Analisando os resultados da última década, o ano de 2010 configurou-se 


\section{ciência
purala}

como o de maior mortalidade (12,03\%), seguido por 2013 (11,80\%) e 2011 (10,81\%), enquanto nos anos de 2018 e 2019 observou-se uma redução dos índices quando comparados ao período antecedente (Figura 1).

Figura 1: Distribuição dos óbitos maternos no estado da Bahia, Brasil, 2010 a 2019. Salvador-BA, Brasil, 2020.

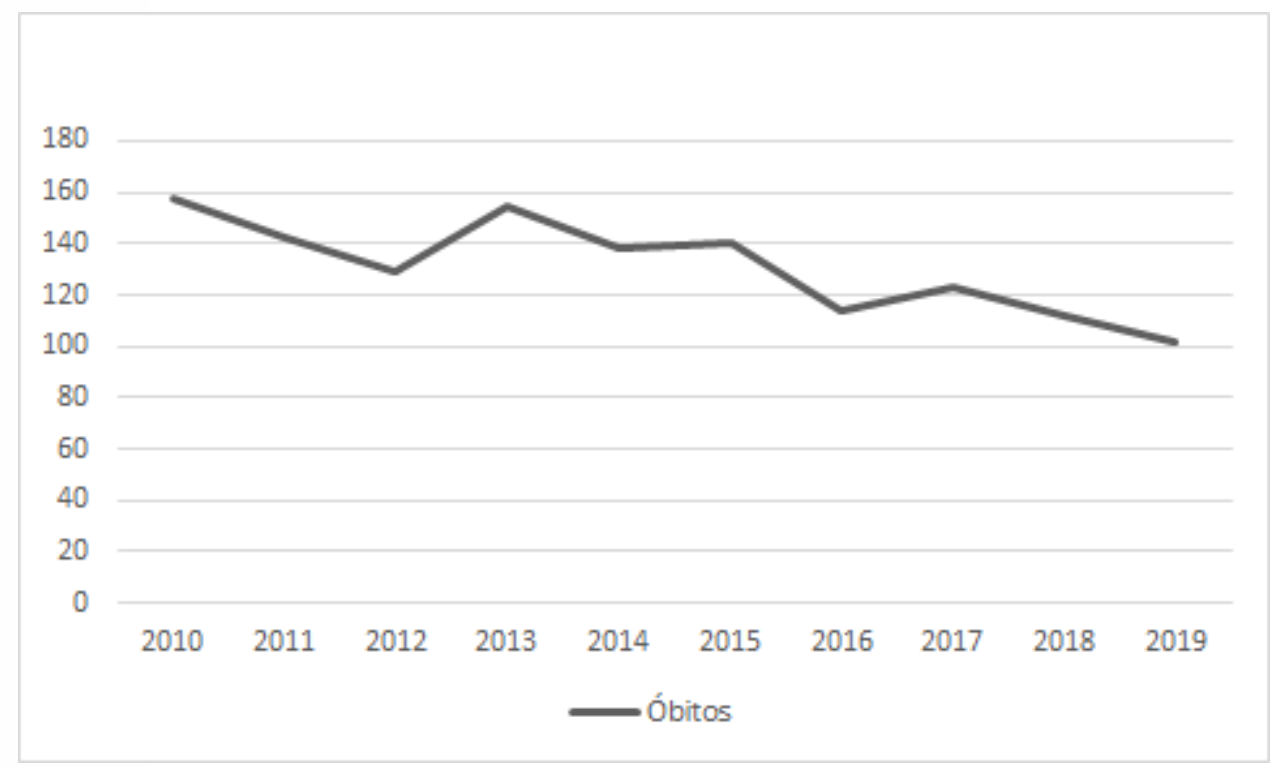

Fonte: Sistema de Informação sobre Mortalidade - SIM (2020)

Quanto ao perfil de mulheres baianas que foram a óbito durante a gestação ou no puerpério, observou-se que àquelas pertencentes à faixa etária de 30 a 39 anos $(44,35 \%)$, seguida por mulheres entre 20 a $29(39,11 \%)$ e com idade 40 a 49 anos $(11,69 \%)$ foram as que mais morreram. Em relação à raça/cor, notou-se que a mortalidade materna acomete principalmente mulheres não brancas $(81,11 \%)$, enquanto que as brancas representam menor percentual das vítimas (12,11\%). Cerca de 6,78\% tiveram essa informação ignorada no preenchimento do documento de óbito. Quanto às causas de falecimento, 1261 notificações continham essa informação dentro do período analisado, onde, os óbitos por causas diretas representaram $66,61 \%$ e as indiretas 33,39\%. Dentre as causas diretas, a hipertensão (19,67\%), hemorragia $3,40 \%)$ e infecção puerperal $(4,68 \%)$ foram elencadas como principais motivações, quanto que, o agravamento de doenças do aparelho circulatório $(9,20 \%)$ e a AIDS $18 \%$ ) configuraram-se como as principais causas dos óbitos pelas causas indiretas, 


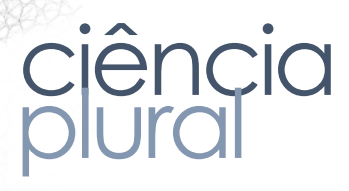

conforme demonstrado na Tabela 1. Analisando-se o local de ocorrência desses eventos, 90,17\% dos óbitos foram registrados no ambiente hospitalar. Em obstantes mortes em domicílio $(2,9 \%)$, outros locais (2,8\%), outros estabelecimentos de saúde (2,5\%) e em via pública $(1,5 \%)$ apareceram em menor proporção.

Tabela 1. Principais causas da mortalidade materna na Bahia de acordo com as notificações. Salvador-BA, Brasil, 2020.

Causa da morte Número total $\quad$ \%

\section{Com causas obstétricas diretas}

Hemorragia

Hipertensão

Infecção puerperal

Causas diretas não especificadas

\section{Causas obstétricas indiretas}

Abortos
50

169

248

59

314

24,90

03,97

13,40

19,67

04,68
15

01,18

116

09,20

Complicações associadas com o aparelho circulatório

Causas indiretas não especificadas

Fonte: Ministério da Saúde - Sistema de Informações sobre Mortalidade

\section{iscussão}

Os principais achados desse estudo apontam que Salvador $(33,74 \%)$, Feira de Santana $(6,24 \%)$ e Vitória da Conquista $(4,80 \%)$ foram os municípios do estado com 


\section{ciêncíncia
puurl}

maior prevalência de mortalidade. Esses resultados podem ser justificados devido a essas cidades se configurarem como polos regionais em saúde para diferentes municípios circunvizinhos, e também pelas limitações relatadas em notificar e alimentar o sistema nos municípios de pequeno porte. Além disso, evidenciam que essas localidades podem possuir fragilidades no que concerne ao acompanhamento gestacional, bem como dificuldades em identificar e monitorar condições que podem predispor a gestante ao risco ${ }^{4}$.

O perfil de mortalidade por faixa etária revelou que mulheres de 30 a 39 anos $(44,35 \%)$ foram as mais acometidas. Esse resultado entra em consonância com estudo realizado por Castro $^{9}$, no Rio de Janeiro, e pode ser justificado pela maior propensão a doenças crônicas pré-existentes, ocasionadas pelo envelhecimento, que contribuem para complicações durante a gestação, e resultam em maior mortalidade materna. Dessa forma, é válido ressaltar a importância de analisar esses fatores que predispõem a mãe ao risco a fim de intervir com ações concretas para reduzir as mortes evitáveis ${ }^{10}$.

A mortalidade entre 20 a 29 anos também demonstrou ser elevada (39,11\%) e chama a atenção por abranger um período etário onde não é esperada mortalidade acentuada, devido à menor ocorrência de doenças crônicas. No entanto, fatores subjacentes como desconhecimento de sinais e sintomas de riscos, e tentativas de abortos devido gestações não planejadas podem contribuir para esse achado ${ }^{11}$. Nesse sentido, observa-se a importância de intervenções preventivas em saúde, especificamente para esse grupo, com medidas que garantam o acesso ao serviço desde o planejamento familiar até o puerpério, como forma de garantir a integralidade em saúde, uma vez que a perda de mulheres de forma prematura reverbera em impactos familiares, sobre as comunidades, e para a economia ${ }^{10}$.

A maior prevalência de mortalidade materna entre mulheres não brancas $(81,11 \%)$ quando comparadas às brancas $(12,11 \%)$ entra em conformidade com estudo ealizado por Moura11, ele verificou que mulheres não brancas apresentam maior revalência de desfechos negativos na gestação, o que pode ser justificado pelas ndições socioeconômicas menos favoráveis e pelo maior percentual de 
analfabetismo, fatores que repercutem na dificuldade de entendimento das orientações fornecidas pelos profissionais de saúde, perpetuando problemas de saúde que tendem a ter cunho social. Entretanto, vale salientar que no estado analisado, a maioria da população é constituída por indivíduos não brancos, o que pode contribuir para o achado 12,13 .

No que diz respeito às principais causas da mortalidade, os resultados apontaram fragilidade no preenchimento dos dados, devido ao elevado percentual de óbitos por causas não especificadas, entrando em conformidade com outros estudos realizados no país que apontam as causas de morte decorrentes de gravidez, parto e puerpério como as que possuem as maiores lacunas nas Declarações de Óbito ${ }^{14}$, o que desencadeia uma série de prejuízos ao Sistema de Saúde, uma vez que as subnotificações comprometem o conhecimento das comorbidades e seus desfechos ${ }^{15}$.

Dentre as causas de óbitos notificados, as que apresentaram maior relevância foram aquelas associadas às causas diretas do período gestacional $(66,61 \%)$. Este achado revela-se importante, visto que esse desfecho pode estar relacionado à qualidade de assistência à saúde da mulher ${ }^{16}$. Além disso, alguns estudos reportaram outras motivações que contribuem para o incremento desse tipo de óbito, a saber: síndrome hemorrágica, doença hipertensiva específica da gestação, transtorno de placenta e aborto ${ }^{17,18}$.

No estado da Bahia, a hipertensão foi elencada como principal agravo por causa direta $(19,67 \%)$, seguindo uma tendência de estudos conduzidos tanto no âmbito nacional quanto internacional, cujos resultados apontam o aumento da pressão arterial durante a gestação como um dos mais importantes fatores que predispõem a complicações severas, como falência de órgãos vitais maternos e mortalidade tanto materna quanto fetal. Nesse sentido, durante o pré-natal, medidas como o acompanhamento dos níveis pressóricos, utilização de anti-hipertensivos e a adoção de um estilo de vida saudável devem ser estimulados a fim de se obter uma stabilização pressórica ${ }^{11,19}$.

A hemorragia $(13,40 \%)$ e infecção puerperal (4,68\%) também foram elencadas mo causas diretas relevantes. Estudo conduzido em Santa Catarina avaliando os 
óbitos maternos encontrou uma prevalência de $12,42 \%$ dos casos relacionados à hemorragia11, enquanto que, estudo realizado por Medeiros ${ }^{17}$ no estado do Amazonas verificou um percentual de 7,65\% de mortalidade associada à infecção. Os resultados se diferem do presente estudo apontando a necessidade da condução de pesquisas em cada estado do país, com perspectiva de investigar os principais agravos à saúde da mulher, possibilitando a elaboração de medidas de intervenção durante o planejamento gestacional, gestação, parto e puerpério.

As causas de mortes indiretas ocasionadas em menor proporção $(33,39 \%)$ estão associadas a comorbidades pregressas à gestação ou àquelas que se desenvolveram neste período, não sendo provocadas por causas obstétricas diretas, mas agravadas pelos efeitos fisiológicos da gravidez ${ }^{20}$. No estado, as complicações de doenças do aparelho circulatório $(9,2 \%)$ e a AIDS $(1,18 \%)$ foram apontadas como principais, o que entra em conformidade com estudos conduzidos no país que também as apontam como relevantes ${ }^{16}, 21,22$. No que diz respeito às complicações das doenças cardiovasculares, a literatura evidencia maior predisposição de mulheres em apresentarem complicações decorrentes dessa comorbidade tendo entre os desfechos, hospitalizações e morte 23 .

No tocante às complicações decorrentes de infecções pelo vírus da imunodeficiência humana (HIV), notou-se diversos avanços no manejo das mulheres portadoras do HIV acompanhadas e monitoradas pelo SUS, de modo que a qualidade e expectativa de vida foi aumentada em virtude dos novos conhecimentos científicos nessa área de estudo ${ }^{24}$. Além disso, nas últimas décadas, constatou-se uma redução das novas infecções, inclusive as verticais, o que pode estar associado às medidas de educação e prevenção ${ }^{25}$.

Quanto ao lugar onde ocorreram os óbitos, a maioria foi registrada em ambiente hospitalar $(90,17 \%)$, o que pode estar associado ao fato dos hospitais serem uma das principais portas de entrada para realização de partos e para o encaminhamento em sos de complicações associadas à gestação, além de ser um dos locais de referência contra referência da atenção primária à saúde. Resultados semelhantes foram contrados por Castro9. 
Cabe registrar que o presente estudo apresentou limitações devido à utilização de dados secundários do Sistema de Informação sobre Mortalidade, sendo o seu uso dependente da qualidade dos registros pelas unidades notificadoras que podem apresentar incompletude de informações, preenchimento inadequado das variáveis disponíveis no sistema e agregação apenas dos atendimentos do sistema de saúde público brasileiro, o que dificulta analisar se os resultados obtidos podem, de fato, refletir a realidade local de cada município pertencente ao estado. Entretanto, essa utilização apresenta vantagens como, por exemplo, o fato de que os dados são coletados de modo rotineiro, havendo uma padronização no preenchimento das informações que podem ser acessados facilmente graças à informatização do sistema ${ }^{14}$.

\section{Conclusões}

Observa-se por meio deste estudo que a mortalidade materna na Bahia se constitui como um importante agravo em saúde, o que demanda a elaboração de um conjunto de medidas que versem sobre a garantia de intervenções baseadas na ampliação da assistência à saúde, na identificação precoce dos fatores de risco, na educação em saúde, na integralidade, e no preparo da equipe multidisciplinar para atender às gestantes e puérperas do Estado.

Ressalta-se a necessidade de políticas públicas voltadas preferencialmente para o atendimento de gestantes com faixa etária de 30 a 39 anos, não brancas e que apresentem predisposição ao desenvolvimento de fatores de risco associados às causas diretas. Salienta-se a importância do ambiente hospitalar com estrutura adequada para receber as gestantes, e para fornecer um atendimento humanizado e que priorize medidas que não coloquem o direito à vida em risco.

\section{eferências}

Norld Health Organization (WHO). Classificação Estatística Internacional de oenças e Problemas Relacionados à Saúde; 10a Revisão. CBCD, São Paulo; 1995. 
2 Morse ML, Fonseca SC, Barbosa MD, et al. Mortalidade materna no Brasil: o que mostra a produção científica nos últimos 30 anos? Cadernos de Saúde Pública. 2011; 27(4):623-638.

3 Ferraz L, Bordignon M. Mortalidade materna no Brasil: uma realidade que precisa melhorar. Revista Baiana de Saúde Pública. 2013;36(2):527-538.

4 Bonatti AF, Silva AMCD, Muraro AP. Mortalidade infantil em Mato Grosso, Brasil: tendência entre 2007 e 2016 e causas de morte. Ciência \& Saúde Coletiva. 2020;25(1):2821-2830.

5 Oliveira JS, Santos AAP, Santos WB, et al. Mortalidade materna: perfil de um estado do nordeste brasileiro. Research, Society and Development. 2020;9(10): 1-17.

6 Sousa C, Violeta CF. Aspectos históricos da saúde pública no Brasil: uma revisão integrativa. JMPHC| Journal of Management \& Primary Health Care. 2020;12(1):1-17.

7 World Health Organization (WHO), UNICEF, UNFPA, The World Bank. Trends in maternal mortality: 1990 to 2008. Geneva: World Health Organization, 2010.

8 Gois EC, de Jesus CS, da Conceição, et al. Mortalidade materna na Bahia no período de 2012 a 2016. Revista Eletrônica Acervo Saúde. 2019;18(1):1-8.

9 Castro IASG, Andrade M, Silva JL, et al. Mortalidade materna no município do Rio de Janeiro entre os anos de 2015 e 2019: um estudo retrospectivo. Research, Society and Development. 2020; 9(10):1-25.

10 Vega CEP, Soares VMN, Lourenço FNAM. Mortalidade materna tardia: comparação de dois comitês de mortalidade materna no Brasil. Caderno de Saúde Pública. 2017;33( 3 ):1-13.

11 Moura BLA, Alencar GP, Silva ZPD, Almeida MFD. Internações por complicações obstétricas na gestação e desfechos maternos e perinatais, em uma coorte de gestantes no Sistema Único de Saúde no Município de São Paulo, Brasil. Cadernos de Saúde Pública. 2018;34(1):1-13.

12 Nkhwalume L, Mashalla Y. Maternal mortality trends at the Princess Marina and Nyangabwe referral hospitals in Botswana. African health sciences. 2019;19(2):18331840.

Soares VMN, Souza KVD, Azevedo EMMD, et al. Causas de mortalidade materna segundo níveis de complexidade hospitalar. Revista Brasileira de Ginecologia e Obstetrícia. 2012; 34(12):536-543. 
14 Silva BGCD, Lima NP, Silva SGD, et al. Mortalidade materna no Brasil no período de 2001 a 2012: tendência temporal e diferenças regionais. Revista Brasileira de Epidemiologia. 2016;19(1):484-493.

15 Mello Jorge MHPD, Gotlieb SL, Soboll ML, et al. Avaliação do sistema de informação sobre nascidos vivos e o uso de seus dados em epidemiologia e estatísticas de saúde. Revista de Saúde Pública. 1993;27(1):1-46.

16 Rodrigues AGM, Cavalcante AES, Viana AB. Mortalidade materna no Brasil entre 2006-2017: análise temporal. Revista Tendências da Enfermagem Profissional. 2019;11(1):3-9.

17 Medeiros LT, Sousa AM, Arinana LO, et al. Mortalidade materna no estado do Amazonas: estudo epidemiológico. Revista Baiana de Enfermagem. 2018;(32):1-11.

18 Scarton J, Thurow MRB, Ventura J, et al. Mortalidade materna: causas e estratégias de prevenção. Research, Society and Development. 2020;9(5):1-16.

19 Kahuli CN, Nungulo VN, Ayres-de-Campos D. Causas de mortalidade materna em quatro hospitais de referência na Província do Huambo no período de 2011 a 2013. Acta Obstétrica e Ginecológica Portuguesa. 2018;12(4):256-260.

20 Turbeville HR, Sasser JM. Pré-eclâmpsia além da gravidez: consequências em longo prazo para mãe e filho. American Journal of Physiology-Renal Physiology. 2020;318(6):1315-1326.

21 Freitas-Júnior RADO. Mortalidade materna evitável enquanto injustiça social. Revista Brasileira de Saúde Materno Infantil. 2020;20(2):607-614.

22 Soares VMN, Souza KV, Freygang TC, et al. Mortalidade materna por préeclâmpsia/eclâmpsia em um estado do Sul do Brasil. Revista Brasileira de Ginecologia e Obstetrícia. 2009;31(11): 566-573.

23 Leal AF, Lizandro L. Instituições participativas e seus efeitos nas políticas públicas: estudo do Comitê de Mortalidade por Aids de Porto Alegre. Saúde e Sociedade. 2018; 27(1): 94-105.

24 Sobral JPCP, dos Santos JMS, da Silva Araújo RJ, dos Santos TG. A mulher e as doenças cardiovasculares: morbidade hospitalar em Alagoas. Gep News. 2019;3(1):3238.

25. Agostini R, Rocha F, Melo E, Maksud I. A resposta brasileira ao HIV/AIDS em tempos de crise. Ciência Saúde Colet. 2019;24(12):4599-4604. 